\title{
Multi-combination therapy for temozolomide-resistant GBM: identification of temozolomide/small molecule inhibitor combinations that target the MDM2/p53 and PI3K- AKT/mTOR networks
}

Anthony Alfonso ${ }^{1}$, Barbara J. Bailey ${ }^{2}$, Erika A. Dobrota ${ }^{2}$, Nuri Damayanti ${ }^{2}$, M. Courtney Young ${ }^{2}$, Pankita H. Pandya ${ }^{2}$, Aaron Cohen-Gadol ${ }^{3}$, Reza Saadatzadeh ${ }^{2}$, Harlan E. Shannon ${ }^{2}$, and Karen E. Pollok ${ }^{2}$

Indiana University School of Medicine; ${ }^{2}$ Herman B. Wells Center for Pediatric Research, Department of Pediatrics, Section of Pediatric Hematology/Oncology, Riley Hospital for Children at Indiana University Health; ${ }^{3}$ Department of Neurosurgery

Introduction: Glioblastoma (GBM) is the most aggressive malignant brain cancer in adults. Induction of the DNA damage response pathway by Temozolomide (TMZ), a DNA alkylating agent, activates p53 resulting in apoptosis. GBM can adapt by upregulating the pro-survival pathway regulator Protein Kinase B (AKT), which phosphorylates murine double minute 2 (MDM2) resulting in increased MDM2-mediated p53 ubiquitination. We hypothesize that a combination treatment of blood-brain-barrier penetrant small molecule inhibitors (SMIs) to AKT (GDC-0068) and MDM2 protein-protein interaction inhibitor (RG7388), will stabilize p53 expression and potentiate TMZ-mediated effects in a recurrent p53 ${ }^{\text {wt }}$ GBM xenoline.

Methods: Dose response assays followed by Calcusyn statistical analysis determined optimal combination dose ratios. Incucyte imaging analyzed confluence throughout a treatment cycle. Cellular response was characterized by: 1) Western blotting 2) Flow cytometry with SPiDER $\beta-$ Gal and FITC Annexin V/ Propidium lodide to quantify senescent and apoptotic cells, respectively 3) p53 siRNA knockdown to examine p53 dependency in treated cells.

Results: Combination index identified synergism of TMZ in the presence of AKT and MDM2 inhibitors at clinically achievable concentrations. Incucyte confirmed a low-dose triple combination significantly inhibited tumor growth. Western blots detected low expression of cleaved PARP and elevated expression of p53 and p21 in RG7388- treated cells compared to vehicle, suggesting senescence-related growth inhibition. SPiDER $\beta$-Gal and FITC Annexin V/PI assays confirmed a high percentage of senescent cells and minimal apoptosis following combination treatment compared to vehicle or single SMI-treated cells. P53 siRNA knockdown confirmed that cell growth inhibition is p53 dependent in treated cells.

Conclusion: This study provides rationale for targeting p53 in recurrent p53 ${ }^{\text {wt }}$ GBM and reveals that senescence could function as a potential therapeutic resistance mechanism. In future studies, targeting of the MDM2-p53 network in the presence of a SM senescence inhibitor will be evaluated to determine if this increases GBM cell death. 